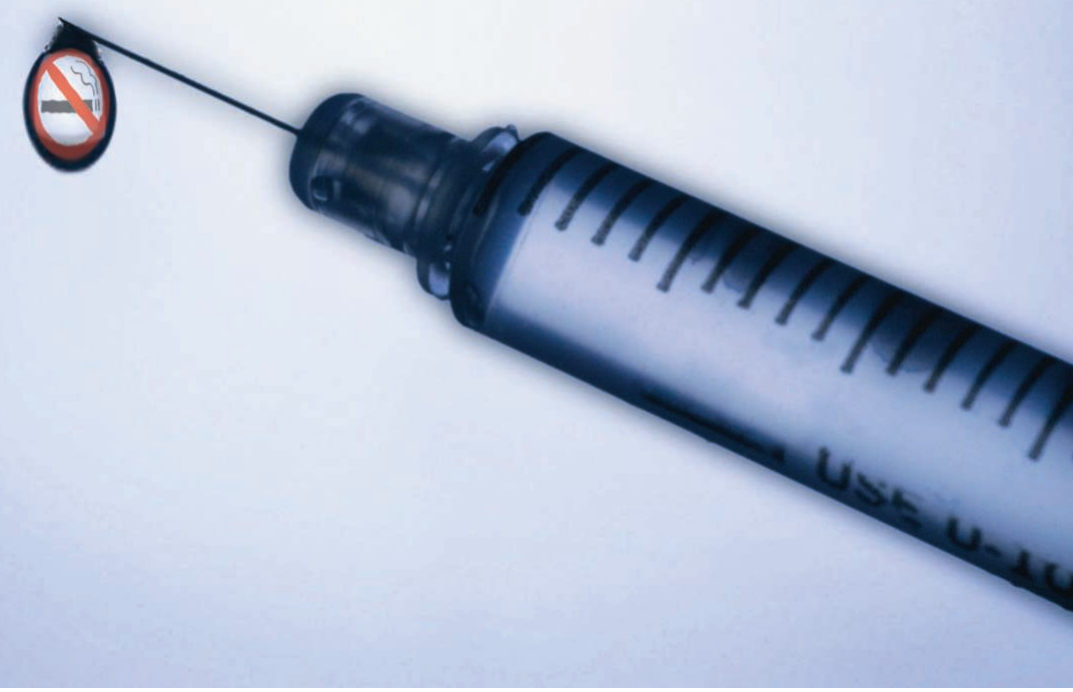

\title{
Chronic Disease Vaccines Need Shot in the Arm
}

Whether vaccines to prevent obesity, asthma, smoking-related illnesses, and other chronic diseases will ever work remains an open question

IN JULY, GLOBAL HEADLINES PROCLAIMED that a "flab jab" was imminent, an obesity vaccine that would allow consumption of endless fast and fatty foods without punishing weight gain. The proof: Mice that were injected with a vaccine targeting the hormone somatostatin gorged on a high-fat diet with significantly less weight gain than those given a sham injection. "Thousands of people contacted us volunteering for clinical trials. Everyone from

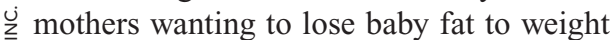
ui lifters in Germany," says vaccinologist Keith Haffer of small, South Dakota-based Braasch Biotech, who led the rodent study.

With funding interest from several South American companies, Braasch Biotech does plan to begin human clinical trials of its somatostatin vaccine late in 2013. Hold the extra cheese on that large sausage pizza, however. It's difficult enough to develop and obtain approval for a traditional vaccine against a bacterium or virus, let alone create one that rouses the immune system to target molecules that drive a chronic disease such as obesity. Indeed, potential vaccines for hypertension, asthma, Alzheimer's disease, obesity, and smoking (because it is a risk factor for heart disease, cancer, stroke, and more) have all been hyped for their promise in recent years and then suffered high-profile failures.

In 2002, for example, a vaccine that raised antibodies to the $\beta$-amyloid protein that accumulates in the brains of people with Alzheimer's disease suffered a scary setback when
$6 \%$ (18 of 298) of the clinical trial subjects receiving the AN1792 vaccine developed a severe brain inflammation. And Nabi Biopharmaceuticals's nicotine vaccine, designed to suck the high out of smoking, failed in a Phase III trial last year. Switzerland-based Cytos Biotechnology also attempted vaccines for smoking, as well as hypertension and type 2 diabetes, and though its researchers made progress, the company ran out of funding in 2011 and abandoned most of those efforts.

Vaccine developers also face a "psychosocial" problem, says Kim Janda, a chemist at the Scripps Research Institute in San Diego, California, who has worked on vaccines for

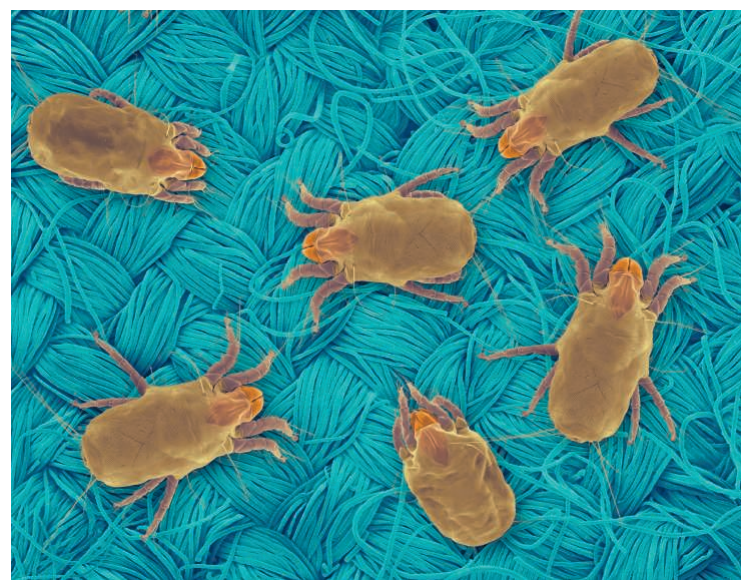

Mite-y vaccine. Dust mites such as Dermatophagoides farinae (pictured) are a trigger of allergies and asthma, prompting efforts to immunize people against one of their proteins. obesity, smoking, and addictive drugs for the past 30 years. "In large part, society still views addiction or even obesity as a moral failure rather than a chronic disease." It's difficult to persuade drug companies and the general population to invest in treating something they view as a failure of willpower with an intervention like a vaccine, Janda says.

But he isn't giving up and neither are others. "If you can find a target that is the underlying cause" of an illness, Janda says, "then you can develop a vaccine for its treatment." There are now a number of vaccines in clinical trials for cancer, which is considered a chronic disease, Janda says, so why not ones for obesity, diabetes, and drug abuse?

"We know why we failed previously and that there are clear pathways in front of us," adds Martin Bachmann, an immunologist formerly with Cytos Biotechnology. He says that poor antibody responses and lack of specificity — critical flaws for a vaccine - are problems that companies are now addressing by using full-length proteins, rather than peptides, and experimenting with viruslike particles that yield a higher and more consistent antibody response.

\section{Mite-y vaccine}

Vaccines were originally developed to combat microbial pathogens such as the smallpox virus and the tuberculosis bacterium; people are traditionally injected with live or dead copies of a pathogenic microbe, or with its molecular components - a viral surface protein, for example - to rally the immune system to produce antibodies or cells that specifically target the invader for destruction.

Yet vaccines may be able to do more than prevent infections. Consider asthma, the target of one vaccine effort. Worldwide, more than 300 million people suffer from asthma, often sparked by a violent immune response to common environmental allergens. Current treatments include corticosteroids, which reduce inflammation but have side effects, and a procedure called desensitization in which asthma/allergy patients are given increasing doses of an allergen cocktail. But the success of desensitization varies from person to person and occasionally causes a life-threatening reaction: anaphylaxis.

A team led by Bruno Pitard at the University of Nantes in France is now tackling asthma with a 
variation on the traditional vaccine. Pitard's strategy stems from the observation that $50 \%$ of Europeans with allergies harbor antibodies against the Der $\mathrm{f} 1$ protein from Dermatophagoides farinae, one of the most common dust mites in the United States and Europe. But instead of immunizing with actual Der $\mathrm{f}$ 1 proteins from this mite, which can trigger an allergic response in people with asthma, Pitard and his colleagues are testing a vaccine composed of DNA coding for the protein, with the idea that it would train the immune system to tolerate it.

When naked DNA is injected into the body, however, it is rapidly degraded before it can express the antigen it encodes. That problem has frustrated many developing naked DNA vaccines, so Pitard is now treating his Der f 1 DNA with so-called tetrafunctional block copolymers, which, as his team discovered in 2009, encourage gene delivery into the skeletal muscle, where the protein can then be manufactured. The team recently immunized mice using this modified vaccine, and those mice had a fivefold reduction in their asthmatic reaction, Pitard says. Lung tissue and bronchioles carried far fewer inflammatory cells and cytokines than in asthmatic mice vaccinated with a placebo. Despite that encouraging data, it will be at least 5 years before a clinical trial of the asthma vaccine begins, Pitard predicts.

Most candidate vaccines for chronic diseases don't target microbial molecules but proteins made by the human body. Take the strategy behind the original Alzheimer's disease vaccine, which sought to activate the immune system against $\beta$ amyloid. Because of the dangerous brain inflammation that followed, companies have largely turned to a so-called passive immunization, an approach in which they create antibodies targeting $\beta$ amyloid outside the body, then inject them (Science, 17 August, p. 790). But several companies are still studying vaccines against different forms of $\beta$ amyloid, which they hope will not produce the same side effects.

The various obesity vaccines also go after natural human proteins. Somatostatin, a small peptide hormone produced in the hypothalamus, inhibits growth hormone and insulin-like growth factor, which increase metabolism. The interest in a somatostatin vaccine for humans began when Haffer was searching for another use for Braasch Biotech's vaccine Somatovac, which they found was a promising way to boost milk production in cows and lean meat production in pigs without using bovine growth hormone or antibiotics. He realized that Somatovac might also promote leanness in humans and therefore fight obesity.

Haffer sent two different versions of the

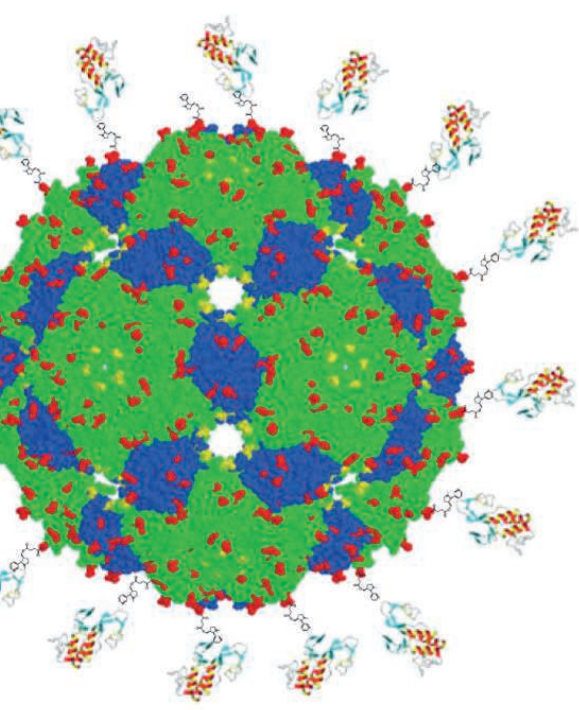

Vaccine recipe. First produce a viruslike particle, then chemically link multiple copies of an antigen, such as nicotine. The result: a conjugate vaccine that triggers strong antibody production.

somatostatin vaccine to Jackson Laboratory in Bar Harbor, Maine, where researchers there tested them on mice that had previously bulked up from consuming a high-fat diet for 8 weeks. Neither vaccine made the alreadyplump rodents lose weight, but they gained $10 \%$ less weight than control rodents, even though all the animals ate the same quantity of high-fat food during the 6-week study, Haffer reported in July in the Journal of Animal Science and Biotechnology.

The concept of creating vaccines for diseases like obesity isn't outlandish, but choosing the target is tricky, says George Jackson of the University of Texas Medical Branch in Galveston, a neuroscientist who is working on a next-generation vaccine for Alzheimer's disease. With Alzheimer's, he points out, you could potentially "target Mr. Hyde without harming Dr. Jekyll" because the culprit-a rogue form of $\beta$ amyloid - appears to have no beneficial role. Obesity is different, he says: "Somatostatin is doing something bad but also something good. A vaccine could cause side effects by interfering with that normal function." Immunizing against an endog- enous hormone controlling appetite might cause anorexia or wasting away, for example.

Haffer isn't the first to dream of an obesity vaccine. Janda and others have targeted the appetite-stimulating hormone ghrelin in their attempts to build an obesity vaccine. Back in 2006, Janda made headlines similar to those received by Haffer's work when he and colleagues reported that vaccinating adult male rats with a segment of ghrelin protein, or the full-length version, slowed weight gain and fat buildup in the body but didn't seem to affect appetite. Janda cautioned at the time that the study rats dined on a mundane, lowfat, low-energy chow. He couldn't predict from this study whether the vaccine would prevent diet-induced obesity - the kind that afflicts many people consuming a high-fat Western diet - or trigger weight loss in animals that were already obese.

Mariana Monteiro, an endocrinologist at the University of Porto in Portugal, presented more evidence at The Endocrine Society's Annual Meeting in June 2011 that an antighrelin vaccine could reduce appetitepromoting brain chemicals in mice. She revealed that tethering ghrelin to a viral protein could trigger enough antighrelin antibodies in the rodents to reduce eating, increase energy use, and reduce levels of neuropeptide $\mathrm{Y}$ - a potent appetite stimulator. But there was no overall weight loss at the end of the study. Monteiro wrote in October 2011 in Expert Review of Vaccines that the underwhelming impact on long-term food intake and body weight "might be due to activation of compensatory mechanisms."

Janda and his team have also explored passive immunization: injecting antighrelin antibodies in mice. In a study published in February in Molecular Pharmaceutics, Janda's team showed that a cocktail of three monoclonal antibodies targeting ghrelin could curb appetite and increase energy use. While this isn't really a ghrelin vaccine because it bypasses the immune system, it similarly "protects" the mice from the hormone and dulls its role as an appetite stimulant.

Even with expanding numbers of overweight and obese people worldwide, Janda's results so far haven't secured him National Institutes of Health funding to further develop a ghrelin vaccine. He's skeptical himself about tackling obesity this way. "I don't think there is one controlling molecule for metabolism," he says. "It's not going to be a panacea for all obesity."

Bachmann suspects that trying to develop a vaccine for obesity is hopeless. "It's so com- 
plicated," he says, "and people love to eat." An obesity vaccine is fighting all the evolutionary safeguards that encourage an animal to eat and ward off starvation. He's also not convinced that mice are a reliable indicator of whether an obesity vaccine will work in humans, noting that "every animal has different feeding behavior."

\section{Nullifying nicotine}

Bachmann remains more optimistic about a potential nicotine vaccine that could help people stop smoking, even though those efforts, too, have stumbled. A nicotine vaccine is subtly different from - and potentially safer than-those targeting obesity-related molecules or Alzheimer's disease protein. The strategy, as with other so-called addiction vaccines for heroin and cocaine, is to create enough antinicotine antibodies in the blood to diminish the amount of the compound that makes it to the brain, in theory making an individual cigarette less appealing. Nicotine isn't normally in the body, so there arguably should be less risk of side effects from inducing antibodies that block it.

In the late 1990s, scientists at Nabi developed and began testing NicVAX, a traditional conjugate vaccine that tethered nicotine, which by itself is so small that it's invisible to the immune system, to a readily detected bacterial protein. Animal studies were promising: The vaccine stimulated the production of antibodies that bound much of the nicotine in the blood before it reached the brain, suppressing the pleasurable nicotine high.

The initial clinical trials examining NicVAX's safety revealed no significant concerns, and by late 2007 Nabi announced that additional Phase II trials had allowed it to zero in on the most effective vaccine dose. The company also found that among the vaccinated smokers, the antibody response correlated closely with the ability to quit and remain abstinent. Late last year, however, the happy ending did not materialize. Nabi announced that its Phase III trial of NicVAX was a failure: There wasn't a significant difference between the percentage of quitters in the NicVAX group and the placebo group.

Others are also finding an anti-smoking vaccine elusive. In 2008, Bachmann and his colleagues at Cytos tested their own nicotine vaccine in 341 smokers in a 6-month randomized, controlled Phase II clinical 崫 trial; 229 were given the vaccine while 112 received the placebo, administered monthly. After 2 months, the number of quitters in the vaccine group was significantly higher than the placebo group, $47 \%$ versus $35 \%$.

But after 6 months, the difference between the two groups was negligible. A closer look at the data offered some hope: The "high responders," the people who produced the highest levels of antinicotine antibody in response to the vaccine, enjoyed the greatest success. Of the high responders, $57 \%$ had abstained from smoking after 6 months, compared with $31 \%$ of the placebo group. At 1 year, the numbers dropped to $41 \%$ and $21 \%$, respectively, which Bachmann calls "statistically and clinically significant." "Only one-third of the patients had a high antibody response," he says. "If we could improve that by a factor of 3 , then we might have a product."

Vaccine development is an expensive game, however, and Cytos ran out of money a year ago. Bachmann has since launched two companies, Areba and Saiba, which focus on vaccines for Parkinson's disease, malaria, and Alzheimer's disease. "I don't have the money to do smoking," he says, though he is still a "great believer" in the nicotine vaccine.

Producing enough antibodies to bind to the 500 micrograms of nicotine in a cigarette before it crosses the blood-brain barrier in 6 to $10 \mathrm{sec}-$ onds is a formidable hurdle. One radical strategy to meet that challenge, described on 27 June in Science Translational Medicine, is more akin to gene therapy than a vaccine. Janda and collaborator Ronald Crystal, a pulmonologist and genetic medicine expert at Weill Cornell Medical College in New York City, injected mice with a virus that travels to the liver, carrying the gene for a monoclonal antibody with a high affinity for nicotine. There, infected cells release antinicotine antibodies into the blood.

"When you give nicotine to mice, they chill out like people," Crystal says. Their blood pressure and heart rate drop by almost half within 25 minutes. Mice that received the team's "vaccine" were unaffected when later given nicotine, running around with no change in blood pressure or heart rate. When Crystal's team analyzed the animals' blood samples, they found that $83 \%$ of nicotine in the serum was bound to the antibodies made by the inserted gene, preventing it from reaching the brain and triggering the dopamine reward system. In the brain, nicotine concentrations were just $15 \%$ of that in untreated control animals.

"It's a second-generation vaccine for addictive molecules. It's more elegant. It produces more antibody," Crystal says, adding that the virus carrying the monoclonal antibody gene is currently being tested in another gene therapy clinical trial, so he's confident of its safety record.

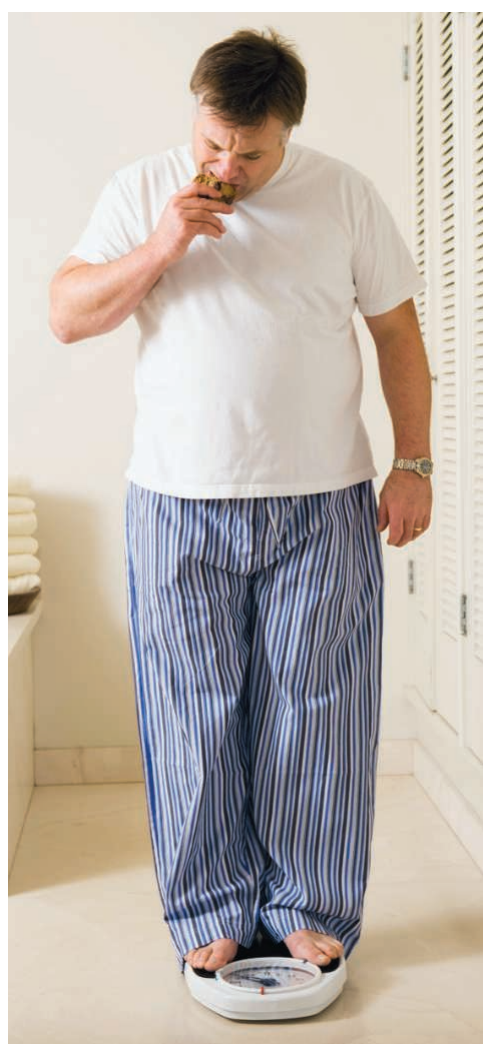

An expanding epidemic. A vaccine that could reduce weight gain would have a domino effect on other chronic conditions such as coronary heart disease, type 2 diabetes, and high blood pressure.
A nicotine vaccine interests Big Pharma, Janda notes, "because there's money in it." Smoking causes about one in five deaths in the United States each year, is responsible for $90 \%$ of lung cancer in men and $80 \%$ in women, and boosts the risk of stroke and heart disease twofold to fourfold.

Pharmaceutical companies may indeed see a big market for a nicotine vaccine, but they're not sure anyone can deliver a safe, effective product. "Novartis has made some contracts with biotech companies to develop antismoking vaccines," says Rino Rappuoli, global head of vaccines research for Novartis Vaccines and Diagnostics in Siena, Italy, "but there has not been much progress. Big companies rely on biotechs to derisk the sector." As the stumbling efforts to immunize people from Alzheimer's, obesity, and smoking attest, however, developing vaccines for chronic diseases remains a risky proposition.

-BIJAL TRIVEDI

Bijal Trivedi is a freelance writer in Washington, D.C. 